\title{
Adverse events and survival with postpericardiotomy syndrome after surgical aortic valve replacement
}

Joonas Lehto, MD, PhD, ${ }^{\mathrm{a}}$ Jarmo Gunn, MD, PhD, ${ }^{a}$ Rikhard Björn, MD, ${ }^{\text {a }}$ Markus Malmberg, MD, PhD, ${ }^{\mathrm{a}}$ K. E. Juhani Airaksinen, MD, PhD, ${ }^{a}$ Ville Kytö, MD, PhD, MSocSc, ${ }^{\text {a, }}{ }^{2}$ Tuomo Nieminen, MD, PhD, ${ }^{c, d}$ Juha E. K. Hartikainen, MD, PhD, ${ }^{\mathrm{e}}$ Fausto Biancari, $\mathrm{MD}, \mathrm{PhD},{ }^{\mathrm{a}, \mathrm{f}}$ and Tuomas O. Kiviniemi, MD, PhD, FESC ${ }^{\mathrm{a}, \mathrm{g}}$

\section{ABSTRACT}

Objectives: Postpericardiotomy syndrome (PPS) is a relatively common complication after cardiac surgery. However, long-term follow-up data on the adverse events and mortality of PPS patients requiring invasive interventions are scarce.

Methods: We sought to assess the occurrence of mortality, new-onset atrial fibrillation (AF), cerebrovascular events, and major bleeds in PPS patients requiring medical attention in a combination database of 671 patients who underwent isolated surgical aortic valve replacement with a bioprosthesis $(n=361)$ or mechanical prosthesis $(n=310$ ) between 2002 and 2014 (Cardiovascular Research Consortium-A Prospective Project to Identify Biomarkers of Morbidity and Mortality in Cardiovascular Interventional Patients [CAREBANK] 2016-2018). PPS was defined as moderate if it resulted in delayed hospital discharge, readmission, or medical therapy because of the symptoms; and severe if it required interventions for the evacuation of pleural or pericardial effusion.

Results: The overall incidence of PPS was $11.2 \%$. Median time to diagnosis was 16 (interquartile range, 11-36) days. Severe PPS was diagnosed in 3.6\% of patients. Severe PPS seemed to be associated with higher mortality (hazard ratio, $2.01 ; 95 \%$ confidence interval, 1.03-3.91; $P=.040$ ). Moderate or severe PPS increased the risk of new-onset AF during the early postoperative period (hazard ratio, 1.72; $95 \%$ confidence interval, 1.12-2.63; $P=.012$ ). No significant associations were found between PPS and cerebrovascular events or major bleeds during the follow-up.

Conclusions: Patients with PPS requiring invasive interventions are at increased risk for mortality unlike those with mild to moderate forms of the disease. PPS requiring medical attention is associated with a higher AF rate during the early postoperative period but has no significant effect on the occurrence of major stroke, stroke or transient ischemic attack, or major bleeds during long-term follow-up. (J Thorac Cardiovasc Surg 2020;160:1446-56)

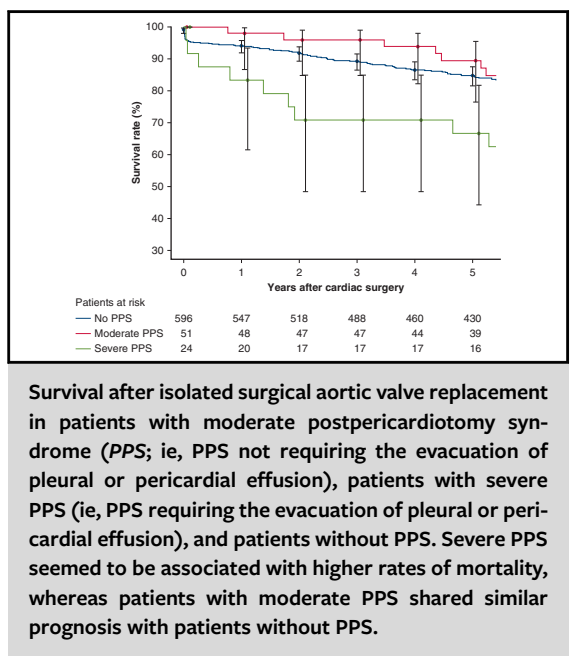

CENTRAL MESSAGE

Patients with severe PPS were at increased risk for mortality. A transient increased risk of new-onset AF was observed, but PPS had no effect on the occurrence of cerebrovascular events or major bleeds.

\section{PERSPECTIVE}

There is a clinical need for more intensive followup in patients with severe PPS. Severe PPS should be considered as a separate end point in the future clinical trials to identify the factors and treatment methods that affect mortality and to improve patient care and recovery.

See Commentaries on pages 1457 and 1458.
From the ${ }^{\mathrm{a}}$ Heart Center, Turku University Hospital and University of Turku, Turku, Finland; ${ }^{b}$ Research Center of Applied and Preventive Cardiovascular Medicine, University of Turku, Turku, Finland; ${ }^{c}$ Department of Internal Medicine, Helsinki University Hospital and University of Helsinki, Helsinki, Finland; ${ }^{\mathrm{d}}$ Department of Internal Medicine, South Karelia Central Hospital, Lappeenranta, Finland; ${ }^{\mathrm{e}}$ Heart Center, Kuopio University Hospital, Kuopio, Finland; ${ }^{\mathrm{f}}$ Department of Surgery, Oulu University Hospital and University of Oulu, Oulu, Finland; and ${ }^{\mathrm{g} D i v i-}$ sion of Cardiovascular Medicine, Brigham and Women's Hospital and Harvard Medical School, Boston, Mass.

This study was funded by the Finnish Medical Foundation, the Finnish Foundation for Cardiovascular Research, Helsinki, Finland; State Clinical Research Fund (EVO) of Turku University Hospital, Turku, Finland; the Emil Aaltonen Foundation; the
Maud Kuistila Foundation; and an unrestricted grant from Bristol-MyersSquibb-Pfizer.

Received for publication Aug 19, 2019; revisions received Dec 29, 2019; accepted for publication Dec 31, 2019; available ahead of print Jan 28, 2020.

Address for reprints: Joonas Lehto, MD, PhD, Heart Center, Turku University Hospital, POB 52, FI-20521 Turku, Finland (E-mail: jojuleh@utu.fi). $0022-5223$

Copyright (C) 2020 The Author(s). Published by Elsevier Inc. on behalf of The American Association for Thoracic Surgery. This is an open access article under the CC BY-NC-ND license (http://creativecommons.org/licenses/by-nc-nd/4.0/). https://doi.org/10.1016/j.jtcvs.2019.12.114 

Abbreviations and Acronyms
$\mathrm{AF} \quad=$ atrial fibrillation
CI = confidence interval
HR = hazard ratio
NYHA $=$ New York Heart Association
PCI = percutaneous coronary intervention
PPS = postpericardiotomy syndrome
SAVR $=$ surgical aortic valve replacement
TIA $=$ transient ischemic attack

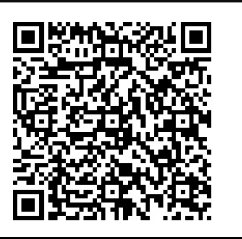

Postpericardiotomy syndrome (PPS) is a relatively common complication after cardiac surgery. ${ }^{1}$ PPS is known to cause prolonged hospital stay, readmissions, and invasive interventions, including the evacuation of pleural and/or pericardial effusion, ${ }^{2-6}$ but its prognosis is considered to be benign. ${ }^{1}$ However, a recently published large epidemiological study showed an association between PPS and mortality during the first year after the surgery, ${ }^{7}$ challenging the presumed benign nature of the syndrome. Furthermore, it is unclear whether PPS is associated with other postoperative adverse events that could also mediate the higher mortality, such as newonset atrial fibrillation (AF), cerebrovascular events, and major bleeding. The aim of this real-world study was to evaluate the effect of PPS on late adverse events after surgical aortic valve replacement (SAVR).

\section{METHODS \\ Data Collection}

The present analysis includes patients from 2 separate databases included in the Consortium Aiming at Reducing Adverse Outcomes Related to Atrial Fibrillation in Patients Undergoing Cardiac Procedures (CAREFIB) Atrial Fibrillation, Stroke, and Bleeding in Patients Undergoing Aortic Biovalve Implantation (CAREAVR); ClinicalTrials.gov identifier: NCT02626871 and the Cardiovascular Research Consortium-A Prospective Project to Identify Biomarkers of Morbidity and Mortality in Cardiovascular Interventional Patients (CAREBANK); ClinicalTrials.gov identifier: NCT03444259. The consort diagram is outlined in Figure 1. The CAREFIB Consortium is part of a broader ongoing protocol in Finland to evaluate thromboembolic and bleeding complications related to AF management in patients who undergo cardiac procedures. ${ }^{8,9}$

The CAREAVR is a Finnish multicenter retrospective study on the rate of AF, thromboembolic complications, and bleeding events in patients who undergo isolated bioprosthetic and mechanical SAVR. Clinical data on consecutive patients who underwent isolated bioprosthetic SAVR were retrieved from the cardiac surgery units of 3 Finnish university hospitals
(Turku, Oulu, and Kuopio) over the period of 2002 to 2014. Data on consecutive patients who underwent isolated mechanical SAVR were retrieved from Turku University Hospital during the same period. To obtain reliable and accurate follow-up data, only patients from the hospitals' catchment areas were included in this study. All major adverse events including PPS, AF, cerebrovascular events, bleeding, and myocardial infarctions are treated almost exclusively in tertiary health care, and therefore, the patient follow-up for adverse events can be considered reliable. An independent, certified third-party data monitor controlled the integrity of the data at each study site.

The CAREBANK is an ongoing Finnish prospective cohort study on the associations between the features of cardiac tissue samples and clinical phenotypes in patients who undergo cardiac procedures. The present study includes the consecutive patients from the CAREBANK study who underwent isolated bioprosthetic or mechanical SAVR at the Turku University Hospital from February 2016 to July 2018. As part of the study protocol, data on all major adverse events including PPS, AF, cerebrovascular events, and myocardial infarction are collected from the hospital records and by follow-up phone calls at $3,12,24$, and 60 months.

All patient records were individually reviewed using a standardized structured data collection protocol for pre- and perioperative data, discharge data, and long-term follow-up events. The end points of this prespecified study included the occurrence of PPS, postoperative AF during index hospitalization and after discharge, major bleeding episodes, cerebrovascular events, and death.

The causes of death were derived from Statistics Finland. This governmental office monitors the time and causes of death in Finland. Personal and immutable social security numbers are used to identify the patients. Therefore, each case is carefully monitored even if the person moved.

During the study period, the routine anticoagulation practice was to administer enoxaparin 40 to $60 \mathrm{mg}$ subcutaneously once a day starting in the evening of the day of the surgery and continuing until vitamin $\mathrm{K}$ antagonist treatment (started on the first postoperative day) reached the therapeutic level (international normalized ratio $\geq 2.0$ or $\geq 2.5$ ). In the bioprosthetic SAVR patients, anticoagulation with vitamin $\mathrm{K}$ antagonist up to 3 months was a common practice (target international normalized ratio of 2.0-3.0).

PPS was defined by the presence of at least 2 of the following criteria: (1) fever without alternative causes, (2) pericarditic or pleuritic chest pain, (3) pericardial or pleural rubs, (4) evidence of pericardial effusion, and/or (5) evidence of pleural effusion with elevated C-reactive protein level. ${ }^{10}$ The laboratory tests, imaging, and other examinations for differential diagnostics were executed as clinically indicated. Relapse was defined as worsening pericardial or pleural effusion during treatment with medication or after withdrawal of medication. PPS was categorized into 2 subgroups according to the severity of the syndrome: moderate PPS that did not require invasive intervention (ie, the evacuation of pleural or pericardial effusion), and severe PPS requiring invasive interventions such as pericardial or pleural drainage.

Diabetes, dyslipidemia, and hypertension were defined as a disease requiring medical treatment and chronic lung disease as a pulmonary disease requiring the long-term use of bronchodilators or steroids. Peripheral arterial disease was defined as 1 or more of the following: claudication, carotid artery disease of $>50 \%$ diameter, and previous or planned intervention on the abdominal aorta, limb arteries, or carotids. Heavy alcohol consumption was defined as $>14$ doses a week for women and $>21$ doses a week for men. Previous cardiac surgery was defined as 1 or more previous major cardiac operation involving opening of the pericardium. Urgent operation was defined as an operation performed during the index hospital stay, emergency operation as an operation before the next working day, and salvage procedure as an operation for which patients require cardiopulmonary resuscitation en route to the operating theater or before the induction of anesthesia. Major stroke was defined as a permanent focal neurological deficit adjudicated by a neurologist and confirmed via computed tomography or magnetic resonance imaging other than lacunar type $(<20 \mathrm{~mm}$ in 


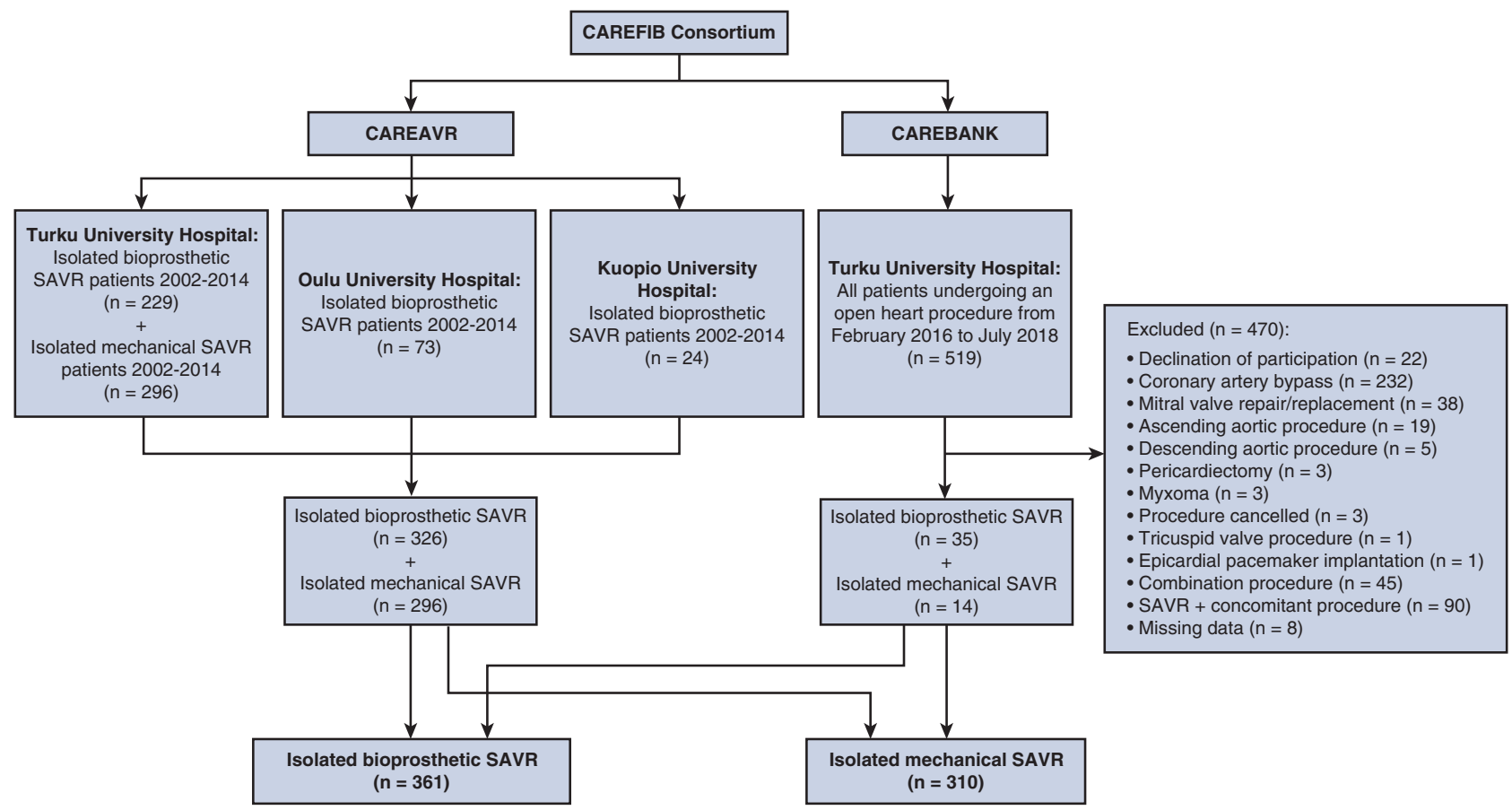

FIGURE 1. Study population consort diagram. CAREFIB, Consortium Aiming at Reducing Adverse Outcomes Related to Atrial Fibrillation in Patients Undergoing Cardiac Procedures; CAREAVR, Atrial Fibrillation, Stroke, and Bleeding in Patients Undergoing Aortic Biovalve Implantation; CAREBANK, Cardiovascular Research Consortium-A Prospective Project to Identify Biomarkers of Morbidity and Mortality in Cardiovascular Interventional Patients; $S A V R$, surgical aortic valve replacement.

diameter). Transient ischemic attack (TIA) was defined as a transient ( $<24$ hours) focal neurological deficit adjudicated by a neurologist. The combination end point stroke or TIA includes all ischemic strokes (lacunar and major) combined to TIAs. Major bleed was defined as an overt, actionable sign of hemorrhage that requires diagnostic studies, hospitalization, or treatment by a health care professional (The Bleeding Academic Research Consortium types 2-5). ${ }^{11}$

The study protocols were approved by the Medical Ethics Committee of the Hospital District of Southwest Finland (numbers 8/1802/2014 and 65/ 1801/2015 from the Ethical Committee of Hospital District of Southwest Finland) and the ethics committee of the National Institute for Health and Welfare (Finland). An informed consent was obtained from the participants of the CAREBANK study. Because of the retrospective, observational nature of the CAREAVR study, an informed consent was not required. The studies conform to the Declaration of Helsinki.

\section{Statistical Analysis}

Statistical analyses were conducted with $\mathrm{R}$ software version 3.5.3 (https://www.R-project.org). Continuous variables were reported as mean \pm standard deviation if normally distributed and as median (25th75 th percentiles) if skewed. The data were tested for normal distribution using the Shapiro-Wilk test. Categorical variables were described as counts and percentages. Cox proportional hazards model, unpaired $t$ test, and Mann-Whitney test were used for univariable analysis. The independency of the predictors of PPS was evaluated using a Cox regression model with interaction terms. The multivariable Cox regression models were performed by including variables of relevance with a $P$ value $<.10$ in the univariable analyses in addition to the prosthesis type. Proportional hazards assumption was assessed using graphical methods. Cause-specific competing risk hazard models accounting for death were used for analyses of outcomes other than mortality. Multiple testing correction was not applied due to the explorative nature of the study.

\section{RESULTS \\ Incidence of PPS}

A total of 671 patients underwent isolated SAVR at the participating hospitals during the study period (bioprosthesis: $\mathrm{n}=361$; mechanical prosthesis: $\mathrm{n}=310$ ). The median follow-up time was 9.0 (6.1-12.0) years for survival and 5.1 (2.4-8.6) years for the other adverse events. Overall, $75(11.2 \%)$ patients developed PPS leading to delayed hospital discharge, readmission, or medical therapy because of the symptoms. The median latency between the operation and medical care contact was 16 (11-36) days. No PPS developed after 188 postoperative days. PPS required the evacuation of pleural effusion in $11(14.7 \%)$, the evacuation of pericardial effusion in $15(20.0 \%)$ patients and led to cardiac tamponade in seven $(9.3 \%)$ patients. Thus, the incidences of moderate and severe PPS were $51(7.6 \%)$ and $24(3.6 \%)$ patients of the total population, respectively.

The baseline characteristics and in-hospital events in patients with and without PPS are detailed in Table 1. No differences were detected between the groups except that patients with PPS were more often male, were more often diagnosed with postoperative pneumonia, and underwent 
TABLE 1. Baseline characteristics and in-hospital events in patients with and without PPS

\begin{tabular}{|c|c|c|c|}
\hline & $\operatorname{PPS}(\mathbf{n}=75)$ & No PPS $(n=596)$ & $P$ value \\
\hline Age, $y$ & $72.0[59.5-77.0]$ & $70.0[60.0-77.0]$ & .89 \\
\hline Female sex & $22(29.3)$ & $272(45.6)$ & .016 \\
\hline Diabetes & $8(10.7)$ & $89(15.0)$ & .42 \\
\hline Dyslipidemia & $42(56.0)$ & $276(46.5)$ & .13 \\
\hline Hypertension & $55(73.3)$ & $403(67.6)$ & .35 \\
\hline Peripheral arterial disease & $1(1.3)$ & $19(3.2)$ & .38 \\
\hline Coronary artery disease & $21(28.0)$ & $144(24.2)$ & .52 \\
\hline Paroxysmal atrial fibrillation & $7(9.3)$ & $62(10.4)$ & .58 \\
\hline Permanent atrial fibrillation & $6(8.0)$ & $54(9.1)$ & .99 \\
\hline Chronic lung disease & $10(13.3)$ & $83(13.9)$ & .97 \\
\hline Active smoking & 7 (11.7) & $58(12.7)$ & .80 \\
\hline Active or ex-smoker & $26(44.1)$ & $171(37.8)$ & .33 \\
\hline Body mass index & $26.7[24.7-29.5]$ & $27.5[24.3-30.9]$ & .76 \\
\hline Heavy alcohol consumption & $4(14.3)$ & $22(9.0)$ & .39 \\
\hline Previous stroke & $4(5.3)$ & $31(5.2)$ & .99 \\
\hline Previous myocardial infarction & $6(8.0)$ & $27(4.5)$ & .36 \\
\hline Previous percutaneous coronary intervention & $8(10.7)$ & 47 (7.9) & .50 \\
\hline Previous cardiac surgery & $2(2.7)$ & $37(6.2)$ & .32 \\
\hline Active endocarditis & $3(4.0)$ & $28(4.7)$ & .88 \\
\hline Previous endocarditis & $3(4.0)$ & $17(2.9)$ & .63 \\
\hline Previous venous thromboembolism & $2(2.7)$ & $11(1.8)$ & .50 \\
\hline \multicolumn{4}{|l|}{ Pulmonary artery hypertension } \\
\hline Moderate to severe (systolic $\geq 31 \mathrm{~mm} \mathrm{Hg}$ ) & $13(23.6)$ & $120(29.0)$ & .50 \\
\hline Severe (systolic $>55 \mathrm{~mm} \mathrm{Hg}$ ) & $3(5.5)$ & $21(5.1)$ & .75 \\
\hline NYHA class III or more & $34(45.3)$ & $313(52.5)$ & .34 \\
\hline Preoperative eGFR $\left(\mathrm{mL} / \mathrm{min} / 1.73 \mathrm{~m}^{2}\right)$ & $76.0 \pm 22.4$ & $77.0 \pm 20.5$ & 60 \\
\hline Urgent, emergency or salvage procedure & $3(4.0)$ & $64(10.7)$ & .11 \\
\hline \multicolumn{4}{|l|}{ In-hospital events } \\
\hline New-onset atrial fibrillation* & $27(43.5)$ & $192(40.1)$ & .64 \\
\hline Deep wound infection & $1(1.3)$ & $4(0.7)$ & .50 \\
\hline Mediastinitis & $0(0.0)$ & $5(0.8)$ & .99 \\
\hline Pneumonia & $11(14.7)$ & $30(5.0)$ & $<.001$ \\
\hline Delayed ventilation & $8(10.8)$ & $70(11.8)$ & .92 \\
\hline Acute de novo dialysis & $1(1.3)$ & $7(1.2)$ & .78 \\
\hline Reoperation due to bleeding & $9(12.0)$ & $53(8.9)$ & .30 \\
\hline Reoperation due to infection & $1(1.3)$ & $3(0.5)$ & .34 \\
\hline Reoperation due to valve problem & $2(2.7)$ & $5(0.8)$ & .045 \\
\hline Reoperation any & $11(14.7)$ & $59(9.9)$ & .13 \\
\hline Length of hospital stay, $d$ & $8.0[7.0-13.0]$ & $8.0[7.0-10.0]$ & .20 \\
\hline
\end{tabular}

Continuous variables are reported as median [interquartile range] or mean \pm standard deviation. Values in parentheses are percentages. PPS, Postpericardiotomy syndrome; NYHA, New York Heart Association; eGFR, estimated glomerular filtration rate. *Patients with preoperative paroxysmal or chronic atrial fibrillation excluded.

more often a reoperation because of a valve problem during the index hospitalization. In a more detailed analysis, male patients with PPS were significantly younger compared with female patients with PPS (69.0 [55.0-75.0] years vs 77.5 [72.3-80.8] years; $P=.002)$. However, the sex difference in PPS patients was not significantly modified by age (Sex $\times$ Age groups interaction $P=.49$ ). In the multivariable model, male sex (hazard ratio [HR], 2.00; 95\% confidence interval $[\mathrm{CI}], 1.19-3.35 ; P=.008)$ and pneumonia during the index hospitalization (HR, 2.43; $95 \%$ 
CI, 1.26-4.72; $P=.008$ ) remained as independent predictors of PPS. No significant differences were detected in the preoperative medications in patients with and without PPS. Also, the comparison of moderate and severe PPS showed no significant differences except that patients with severe PPS presented more often with a history of preoperative permanent $\mathrm{AF}(5[20.8 \%]$ vs $1[2.0 \%] ; P=.001)$ and heavy alcohol consumption (3 [33.3\%] vs 1 [5.3\%]; $P=.039$ ). None of the patients with postoperative pneumonia and PPS were heavy alcohol users.

\section{Treatment of PPS and Relapses}

First-line medication for PPS was glucocorticoids in $64 \%$ of the patients, colchicine in $20 \%$ of the patients, and nonsteroidal anti-inflammatory drugs in $5 \%$ of the patients. Overall $12(16.0 \%)$ of the 75 patients with PPS had a relapse defined by the worsening of pericardial or pleural effusion during treatment with medication or after withdrawal of the medication.

\section{PPS and Mortality}

The median follow-up time for mortality was 9.0 (6.112.0) years and overall 206 deaths occurred during the follow-up. The following were the variables of relevance with a $P$ value $<.10$ in the univariable analyses of mortality: increasing age, female sex, diabetes, hypertension, peripheral arterial disease, coronary artery disease, permanent $\mathrm{AF}$, chronic lung disease, previous myocardial infarction, previous percutaneous coronary intervention (PCI), previous cardiac surgery, New York Heart Association (NYHA) class III or more, lower preoperative estimated glomerular filtration rate, and pulmonary artery hypertension. Previous PCI and previous cardiac surgery were excluded from the multivariable analyses because of their association with previous myocardial infarction. The variables of relevance were similar in the subgroup analyses, except that in the analysis excluding patients who died within 30 days, the variable previous cardiac surgery did not reach the $P$ value $<.10$ required for the multivariable analysis. In addition, in the analysis of the mortality of patients with moderate PPS, previous myocardial infarction and previous cardiac surgery were discarded and active smoking and previous venous thromboembolism were added to the model. Survival after isolated SAVR in patients with severe and moderate PPS separately and in patients with overall PPS, compared with patients without PPS are shown in Figure 2 and Figure 3,D. No association was observed between PPS and all-cause mortality in the overall study cohort. Analysis excluding patients who died within 30 days after the surgery confirmed these results (Table 2). However, patients with severe PPS had increased all-cause mortality (unadjusted analysis: HR, $1.91 ; 95 \% \mathrm{CI}$, $1.01-3.62 ; P=.046$; adjusted analysis: HR, $2.01 ; 95 \% \mathrm{CI}$, $1.03-3.91 ; P=.040)$. The difference in mortality of the severe PPS patients appeared within the first 24 months after the surgery with the median time from the diagnosis to death being 460 (83-1300) days. The survival of patients with moderate PPS was similar to that of patients without PPS (unadjusted analysis: HR, 0.67; 95\% CI, 0.36-1.27; $P=.22$; adjusted analysis: $\mathrm{HR}, 0.70 ; 95 \% \mathrm{CI}, 0.36-1.35$; $P=.29$ ).

The underlying cause of death appearing within 24 months after severe PPS diagnosis $(n=7)$ was often registered as atherosclerotic heart disease or aortic valve stenosis. Only 1 patient had PPS registered as the underlying cause of death and 1 as the intermediate cause of death. The remaining underlying causes of death were single cases of unspecified sepsis, stroke, and falling in the same level eventually leading to pneumonia. Pneumonia was registered as the immediate cause of death in half of the deaths, including the 2 patients with PPS registered as the underlying or intermediate cause of death. No significant baseline differences were found between patients who died within 24 months after severe PPS and patients without severe PPS except that the patients who died within 24 months after severe PPS had more previous myocardial infarction (2 $[29 \%$ ] vs $30[4.6 \%] ; P=.042)$. Preoperative echocardiographic data were available in 6 of 7 patients who died within 24 months after severe PPS diagnosis and in 599 of 647 of patients without severe PPS. The comparison of the 2 groups revealed no significant differences in preoperative aortic valve maximum or mean pressure gradient, aortic valve regurgitation degree, or mitral valve regurgitation degree. However, the patients who died within 24 months after severe PPS had lower preoperative left ventricular ejection fraction compared with patients without severe PPS (median, 48 [43-50] $\%$ vs 61 [51-70] $\% ; P=.014$ ). When patients who died within 24 months after the surgery with and without severe PPS were compared, no significant differences were found in preoperative left ventricular ejection fraction (48 [43-50]\% vs $61[45-73] \% ; P=.116)$ or previous myocardial infarction $(2[29 \%]$ vs $4[8.0 \%]$; $P=.15)$.

\section{PPS and Other Adverse Outcomes}

During the follow-up, a total of $190(35.3 \%)$ patients developed new-onset AF after hospital discharge, 62 $(9.2 \%)$ suffered a major stroke, $111(16.5 \%)$ stroke or TIA, and $107(17.2 \%)$ developed major bleeding. The following were the variables of relevance with a $P$ value $<.10$ in the univariable analyses of new-onset AF: increasing age, male sex, greater body mass index, dyslipidemia, hypertension, chronic lung disease, active or previous smoking, previous stroke, previous myocardial infarction, NYHA class III or higher, lower preoperative estimated glomerular filtration rate, previous endocarditis, and pulmonary artery hypertension. Similarly, the following variables were associated with higher major 


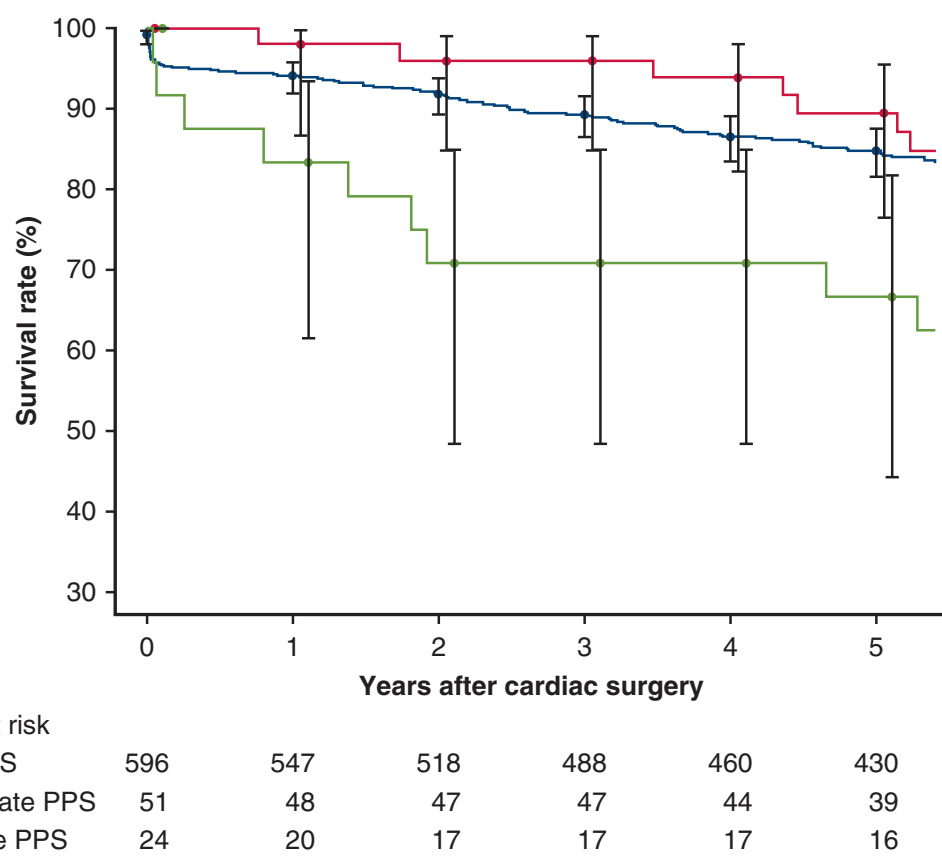

FIGURE 2. Survival after isolated surgical aortic valve replacement in patients with moderate postpericardiotomy syndrome (PPS; ie, PPS not requiring the evacuation of pleural or pericardial effusion), patients with severe PPS (ie, PPS requiring the evacuation of pleural or pericardial effusion), and patients without PPS. Severe PPS seemed to be associated with higher rates of mortality, whereas patients with moderate PPS shared similar prognosis with patients without PPS.

stroke rate and entered to the multivariable model: increasing age, diabetes, hypertension, paroxysmal AF, permanent $\mathrm{AF}$, chronic lung disease, peripheral arterial disease, and NYHA class III or higher. The following were the variables of relevance with a $P$ value $<.10$ in the univariable analyses of stroke or TIA: increasing age, diabetes, hypertension, paroxysmal $\mathrm{AF}$, permanent $\mathrm{AF}$, chronic lung disease, peripheral arterial disease, heavy alcohol consumption, previous stroke, and NYHA class III or higher. Last, the following variables were associated with higher bleeding rate and entered to the multivariable model: younger age, paroxysmal AF, permanent $\mathrm{AF}$, heavy alcohol consumption, previous PCI, previous cardiac surgery, and pulmonary artery hypertension. The Cox proportional hazards univariable models and multivariable adjusted Cox regression models of the effect of PPS on adverse outcomes are detailed in Table 2. PPS had no significant effect on these adverse outcomes (Figures 3 and 4), apart from new-onset $\mathrm{AF}$ in the multivariable analysis. As depicted in Figure 4, this effect appeared within 1 month after the surgery. When AF episodes during the index hospitalization were also included, no significant difference in the incidence of new-onset AF was found between patients with and without PPS (HR, 1.17; 95\% CI, 0.83-1.63; $P=.37$ ). Severe PPS was not associated with the higher occurrence of new-onset AF after hospital discharge (HR, 1.23; 95\% CI, 0.55-2.78; $P=.61$ ), major stroke (HR, $0.61 ; 95 \% \mathrm{CI}$, $0.09-4.44 ; P=.63$ ), stroke or TIA (HR, $0.34 ; 95 \% \mathrm{CI}$,
$0.05-2.46 ; P=.29$ ), or major bleeding (HR, $1.67 ; 95 \%$ CI, 0.61-4.54; $P=.32$ ).

\section{DISCUSSION}

\section{Main Findings}

The main findings of the present study are as follows. (1) The higher mortality of PPS patients seems to relate only to the patients who require invasive interventions because of the syndrome, and (2) PPS requiring medical attentioneither in moderate or severe form - had a significant transient effect on the occurrence of new-onset AF during the early postoperative period, but it did not increase major stroke, stroke or TIA, or major bleeds during long-term follow-up, indicating that the higher mortality risk is attributed to other than these outcomes.

\section{Mortality}

Strikingly, severe PPS resulted in a twofold mortality risk. In contrast, the survival of patients with moderate PPS was similar to that of patients without PPS. The effect of PPS on mortality is illustrated in Figure 5. The higher mortality rate associated with PPS was first reported in a recent large epidemiological study, ${ }^{7}$ but the reasons for this finding remained unknown. Because of the delay of several months between PPS diagnosis and death, the higher mortality rate has been presumed to be related to the underlying immunological changes caused by or resulting in PPS. ${ }^{7}$ In the present study, the delay between severe PPS 


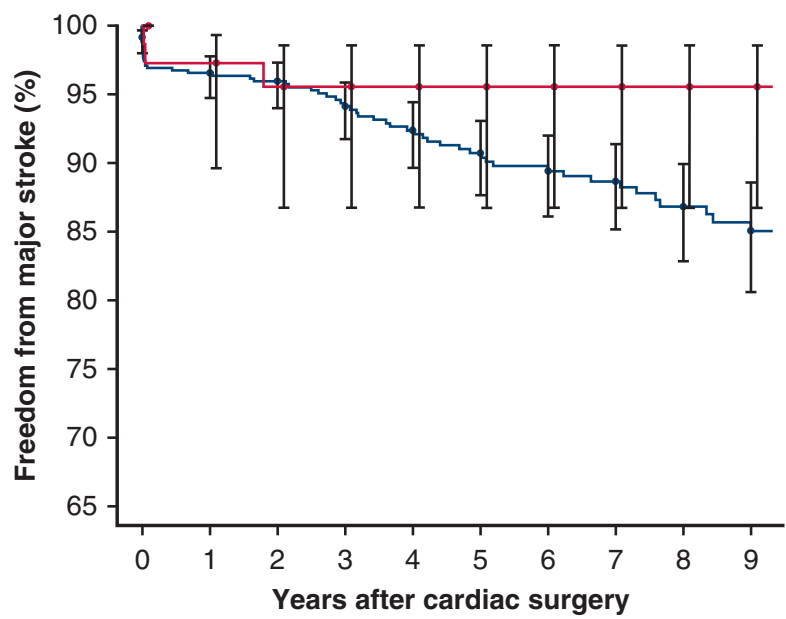

Patients at risk

- PPS - $\begin{array}{llllllllll}596 & 513 & 457 & 394 & 350 & 298 & 249 & 211 & 167 & 134\end{array}$

$\begin{array}{lllllllllll}-\mathrm{PPS}+ & 74 & 61 & 53 & 48 & 38 & 31 & 25 & 18 & 15 & 10\end{array}$

A

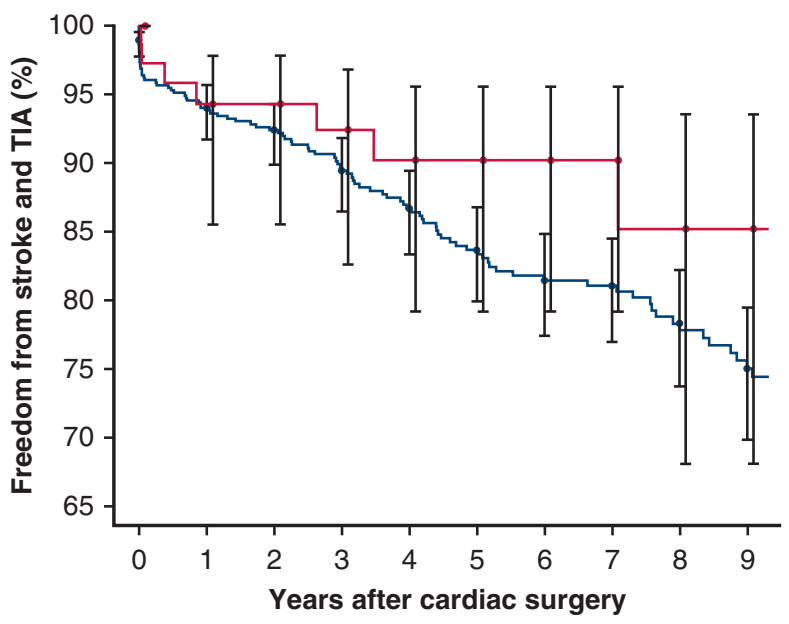

Patients at risk

— PPS - $\begin{array}{llllllllll}595 & 498 & 439 & 374 & 329 & 279 & 231 & 196 & 157 & 126\end{array}$

B

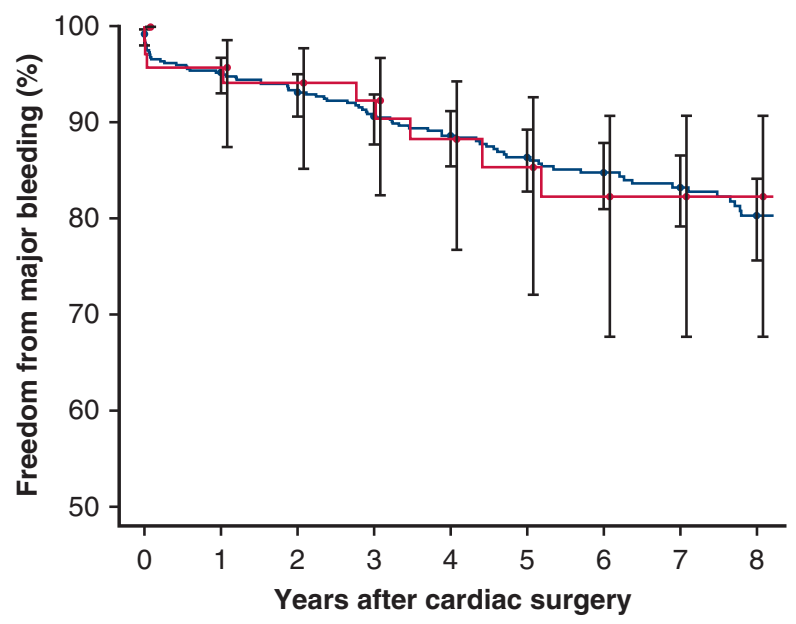

Patients at risk

- PPS - $\begin{array}{lllllllll}550 & 478 & 432 & 382 & 334 & 282 & 233 & 194 & 155\end{array}$

$\begin{array}{llllllllll}-\mathrm{PPS}+ & 71 & 60 & 53 & 48 & 37 & 29 & 21 & 17 & 14\end{array}$

Patients at risk

— PPS - $\begin{array}{llllllllll}596 & 547 & 518 & 488 & 460 & 430 & 363 & 309 & 268 & 215\end{array}$

- PPS + $\begin{array}{llllllllll}75 & 68 & 64 & 64 & 61 & 55 & 45 & 33 & 24 & 20\end{array}$

C

FIGURE 3. Freedom from (A) major stroke, (B) stroke and transient ischemic attack (TIA), (C) major bleeding, and (D) death in patients with (PPS+) and without (PPS-) PPS. PPS requiring medical attention had no significant effect on the occurrence of these adverse events during long-term follow-up. $P P S$, Postpericardiotomy syndrome.

and death varied from weeks to years with a median of 15 months, and the deaths associated with severe PPS took place within 24 months after the syndrome (Figure 2). Considering the causes of death, the higher mortality is most likely caused by the incremental disease burden of PPS and its sequelae, and especially the required interventions. Therefore, in the light of the present results, it is unlikely that PPS itself is the cause of incremental deaths after cardiac surgery. Instead, PPS is likely to complicate previously weak and vulnerable patients, thus increasing mortality with a delay. This hypothesis is supported by the fact that the patients who died within 24 months after severe PPS had somewhat more previous myocardial infarctions and lower preoperative ejection fraction. However, although the relationship with the underlying immunological changes related to PPS seems unlikely, further studies are needed to rule it out completely.

Currently, none of the PPS treatments have been shown to reduce postoperative mortality in previous studies. Nevertheless, none of the studies have been powered to 
TABLE 2. The association of PPS with adverse outcomes after isolated surgical aortic valve replacement

\begin{tabular}{|c|c|c|c|c|}
\hline & \multicolumn{2}{|c|}{ Univariable analysis (PPS vs no PPS) } & \multicolumn{2}{|c|}{ Multivariable analysis (PPS vs no PPS) } \\
\hline & HR $(95 \%$ CI) & $P$ value & HR $(95 \%$ CI $)$ & $P$ value \\
\hline AF new onset & $1.46(0.97-2.19)$ & .072 & $1.72(1.12-2.63)$ & .012 \\
\hline Major stroke & $0.45(0.14-1.43)$ & .17 & $0.46(0.14-1.50)$ & .20 \\
\hline Stroke or TIA & $0.60(0.28-1.29)$ & .19 & $0.63(0.29-1.37)$ & .25 \\
\hline Major bleeding & $0.95(0.49-1.82)$ & .87 & $1.02(0.53-1.98)$ & .94 \\
\hline Death & $0.98(0.62-1.56)$ & .95 & $1.03(0.64-1.67)$ & .89 \\
\hline Death (alive after $30 \mathrm{~d}$ ) & $1.06(0.65-1.73)$ & .82 & $1.07(0.64-1.79)$ & .79 \\
\hline
\end{tabular}

PPS, Postpericardiotomy syndrome; $H R$, hazard ratio; $C I$, confidence interval; $A F$, atrial fibrillation; $T I A$, transient ischemic attack.

investigate the possible mortality benefits of the treatments in either the preventive use or the management of PPS. Therefore, it is possible that, for example, the preventive use of colchicine or more aggressive first-line treatment of PPS would reduce postoperative mortality if studied in an adequately sized research setting. Until further knowledge of the possible mortality benefits of the treatment options is achieved, the best course of action is to provide more intensive follow-up and treatment of the PPS patients who require invasive interventions.

\section{PPS and Other Adverse Outcomes}

PPS was not associated with major stroke, stroke or TIA, or major bleeding. However, we found a significant association between PPS and the occurrence of new-onset AF. The difference in the occurrence of AF appeared shortly after the initial hospital discharge (Figure 4). Thus, it is possible that the pericardial irritation caused by PPS provoked AF paroxysms during the first months after the surgery. In that case, the effect is most likely transient, and therefore, it should not affect the overall prognosis of the patients. In fact, of the PPS patients who developed new-onset AF after hospital discharge within the first 60 postoperative days $(n=19)$, only 3 died during the follow-up and the delay between the operation and death was 3.5 to 5.3 years.

\section{Risk Factors for PPS}

In this study, pneumonia during index hospitalization was associated with PPS. In only 1 previous PPS study the incidence of pneumonia during the early postoperative period has been reported. ${ }^{12}$ In the aforementioned study on patients who underwent isolated coronary artery bypass

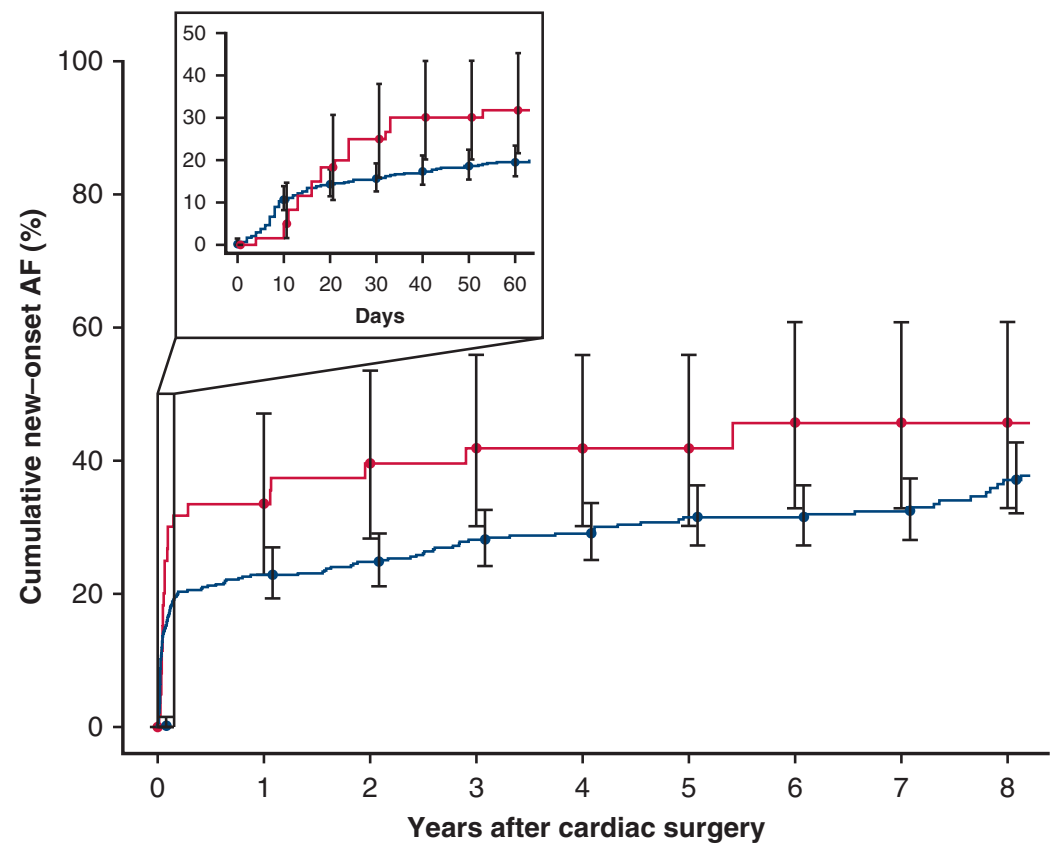

Patients at risk

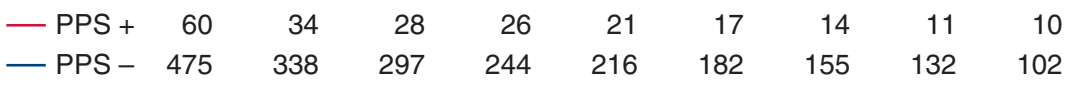

FIGURE 4. Cumulative occurrence of new-onset $\mathrm{AF}$ in patients with (PPS+) and without (PPS-) PPS. PPS appeared to provoke AF paroxysms, and the difference appeared during the first months after the surgery. AF, Atrial fibrillation; PPS, postpericardiotomy syndrome. 


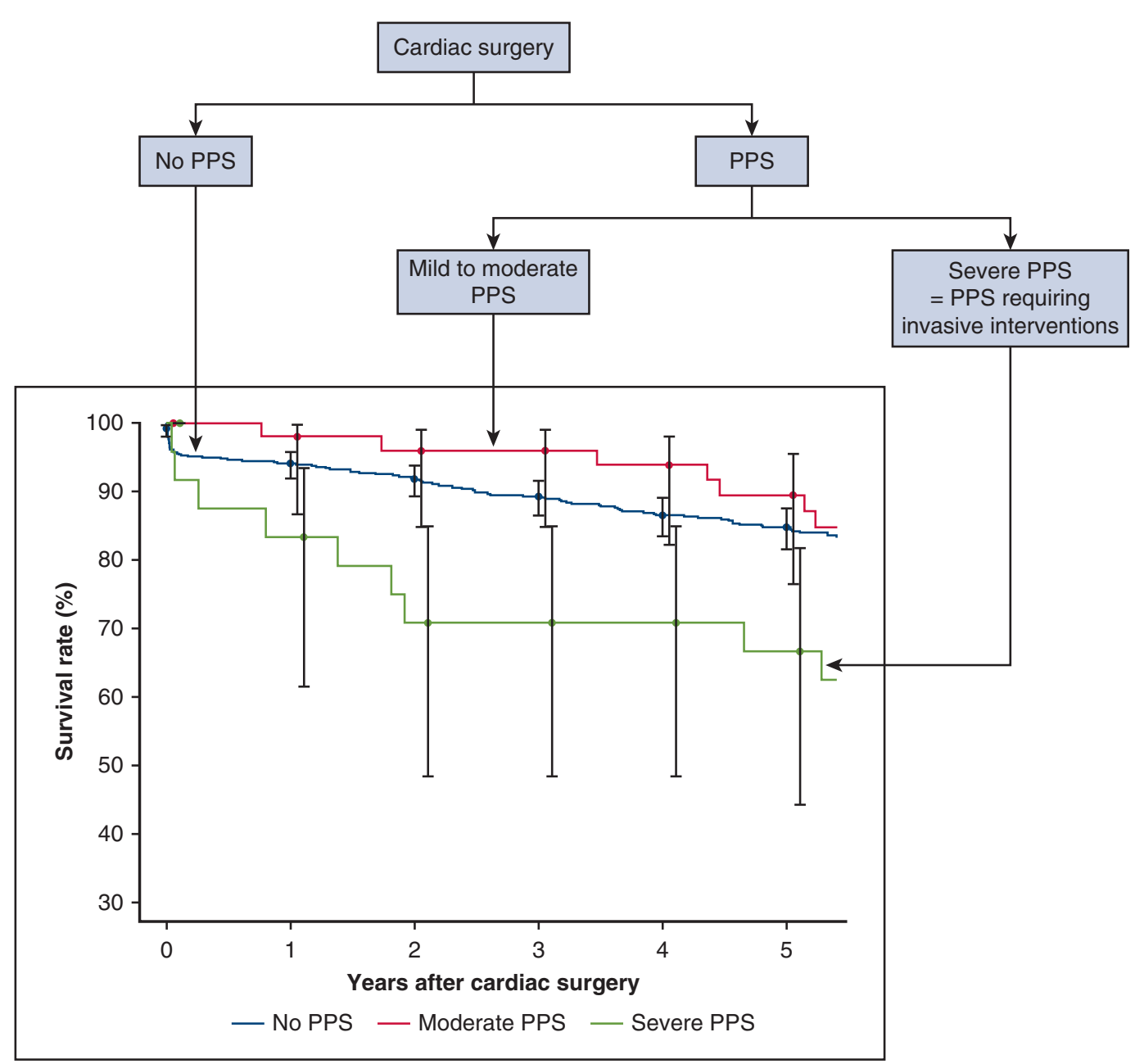

FIGURE 5. Patients with severe PPS (ie, PPS requiring the evacuation of pleural or pericardial effusion) are at increased rates of mortality during the first 24 months after cardiac surgery, whereas patients with mild to moderate PPS and no PPS present equal mortality rates. PPS, Postpericardiotomy syndrome.

grafting, those who developed pneumonia had a trend of higher incidence of PPS $(5.7 \%$ vs $3.4 \% ; P=.39)$. In the present study, the PPS diagnosis after pneumonia during the index hospitalization were typically set within 1 week after hospital discharge. Thus, it is possible that some of the pneumonia were actually early stages of PPS, because pneumonia is one of the most difficult differential diagnoses of PPS during the early postoperative period. This underlines the complexity of diagnosing PPS during the early postoperative period in real life. PPS also typically results in hypoventilation via pleural irritation and pleural effusion, which might expose the patients to the development of pneumonia. However, another possible explanation is that pneumonia causes an immunological activation that exposes the patients to the development of PPS.

Surprisingly, male sex was associated with higher rates of occurrence of PPS. In previous studies, female sex and younger age have been reported as predisposing factors for PPS., ${ }^{2,13,14}$ Male patients with PPS were, however, significantly younger compared with their female counterparts, although the interaction analysis showed no significant interactions between age groups and sex. Both of the previous studies that reported female sex as a risk factor for PPS have included all cardiac surgery types. ${ }^{2,14}$ Besides the multiplicity caused by the variety of procedure types, this results in a markedly wider age distribution compared with the cohort in the present study. Regardless of the negative result in the interaction analysis, it is possible that the effect of male sex is indirect and due to differences in the age distributions of PPS patients in combination with the differences in procedure types. This hypothesis is strengthened by the fact that only $1.0 \%$ of the female patients who underwent surgery were 40 to 49 years of age, which, according to the previous epidemiological study, is the group with the highest difference in PPS occurrence between sexes. ${ }^{14}$ Nevertheless, further studies are needed to achieve a definitive explanation for this finding. 


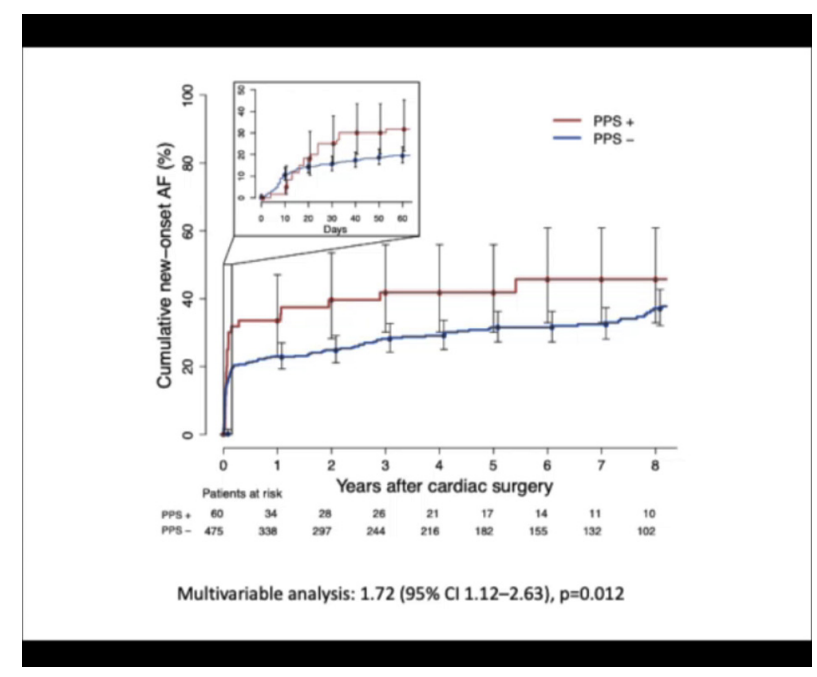

VIDEO 1. Lead author Joonas Lehto explains the key message of the article. Video available at: https://www.jtcvs.org/article/S0022-5223(20) 30231-2/fulltext.

In the present study, PPS patients were most often treated with corticosteroids, although they are no longer recommended because of their association with higher recurrence rates. ${ }^{10}$ However, a large part of the treatment was initiated before the release of the guidelines that recommended not to use corticosteroids as the first-line treatment. Furthermore, the studies that reported the high efficiency of colchicine in the treatment of pericarditis and PPS were mostly published after most of the patients had already undergone the surgery, and all of the patients were receiving vitamin $\mathrm{K}$ antagonist treatment, favoring the use of glucocorticoids instead of high-dose nonsteroidal anti-inflammatory drugs because of the high bleeding risk.

\section{Clinical Implications}

This study has several clinical implications. Patients with severe PPS seem to be at increased risk for long-term mortality. Mortality, however, did not appear to be related to ischemic stroke, or major bleeds, but possibly to pneumonia. Better knowledge of the adverse events of PPS is crucial, because they define the acceptable level of costs and possible side effects of prophylactic methods used. In line with the present results, in a recent study, PPS was associated with all-cause mortality. ${ }^{7}$ Prevention of death requires more detailed information on which PPS patients are at the highest risk. Eventually, this might allow the targeting of possible prophylactic methods and/or effective management options to the patients at the highest risk of death. As pointed out in a previous study, most of the PPS diagnoses reported in earlier prospective studies have been clinically irrelevant. ${ }^{12}$ The higher mortality rate in severe PPS patients supports the view that severe PPS should be considered as an end point in future clinical trials. In addition, besides future research, the classification of PPS into subgroups (moderate and severe) might be useful in the treatment of PPS patients, because it seems to reflect the prognosis of the patients.

\section{Strengths and Limitations}

CAREBANK is a prospective study focused on the adverse events after cardiac surgery. Besides the prospective nature of the database, another strength concerning both of the databases is that a validated, structured case report form was used in data collection. As a quality control of the multicenter CAREAVR database, a professional third party monitored the data. Furthermore, to the authors' knowledge, this is the first study to address the possible adverse events of PPS in a long-term follow-up of several years. The main limitation of this study is the retrospective nature of the CAREAVR data. However, data were obtained from electronic patient records, and data on baseline, operation, and outcomes are reported in detail at each of the participating hospitals. Also, the follow-up was complete for as much as $99.7 \%$ of the patients because the treatment of patients belonging to the catchment areas of the participating institutions is mainly centralized. This allowed us to obtain information on major clinical events requiring hospital treatment and information on outpatient visits after the surgery because they were performed almost exclusively in tertiary health care. Data on late mortality were obtained from Statistics Finland, which ensures the quality of survival data of the patients. The small size is another limitation of this analysis, and therefore, these findings should be viewed as hypothesis-generating. The preoperative echocardiographic data were not available in all patients, and therefore, these factors that possibly affect mortality could not be included in the multivariable analyses. Also, all tests leading to the diagnosis of PPS (eg, chest x-ray and echocardiography) were not performed regularly to all patients (eg, on a weekly basis), so the underdiagnosing of asymptomatic PPS is conceivable. Nevertheless, the tests were performed as clinically indicated, which reflects the "real-world" feature of the present study.

\section{CONCLUSIONS}

The present study suggests that PPS requiring medical attention increases the occurrence of new-onset AF during the early postoperative period but has no effect on major stroke, stroke or TIA, or major bleeding during long-term follow-up. However, severe PPS seems to be the subgroup from which the higher mortality of PPS patients is originated, suggesting that these patients are in the need of more intensive follow-up and treatment (Video 1).

\section{Conflict of Interest Statement}

Dr Lehto has received research grants from Orion Research Foundation, the Finnish Foundation for Cardiovascular 
Research, the Finnish Cultural Foundation, Turku University Foundation, and the Emil Aaltonen Foundation. Dr Gunn has received research grants from Turku University Research Foundation, Turku, Finland, the Clinical Research Fund (VTR) of Turku University Hospital, Turku, Finland, and an unrestricted grant from Vifor Pharma. Dr Malmberg has received research grants from the Clinical Research Fund (VTR) of Turku University Hospital, Turku, Finland, and the Finnish Cultural Foundation. Dr Airaksinen has received research grants from the Finnish Foundation for Cardiovascular Research, the Clinical Research Fund (VTR) of Turku University Hospital, Turku, Finland, lecture fees from Bayer, and Boehringer Ingelheim, and is a member in the advisory boards of Bayer, Astra Zeneca, and Bristol-Myers Squibb-Pfizer. Dr Kytö has received research grants from the Finnish Cultural Foundation, the Clinical Research Fund (VTR) of Turku University Hospital, Turku, Finland, and the Finnish Cardiac Society, and lecture fees from Bayer and AstraZeneca. Dr Nieminen has received research grants from AbbVie, Medtronic, and research funds from Helsinki and Uusimaa Hospital District, and lecture fees from AstraZeneca, Boehringer Ingelheim, Finnish Consulting Group Koulutus, GE Healthcare, Medtronic, Orion, and Sanofi. Dr Hartikainen has received research grants from the Finnish Foundation for Cardiovascular Research, Clinical Research Fund (VTR) of Kuopio University Hospital, Kuopio, Finland, lecture fees from Cardiome AG and Astra Zeneca, and is a member of the advisory boards of Amgen, Pfizer, and Novo Nordisk. Dr Kiviniemi has received lecture fees from Bayer, Boehringer Ingelheim, MSD, Astra Zeneca, St Jude Medical, and Bristol-Myers-Squibb-Pfizer, and research grants from the Finnish Medical Foundation, the Finnish Foundation for Cardiovascular Research, Clinical Research Fund (EVO) of Turku University Hospital, Turku, Finland, Finnish Cardiac Society, the Emil Aaltonen Foundation, the Maud Kuistila Foundation, and an unrestricted grant from Bristol-Myers Squibb-Pfizer, and is a member of the advisory board of Boehringer-Ingelheim, and MSD. All other authors have nothing to disclose with regard to commercial support.
The authors thank study coordinator Tuija Vasankari, RN, for her input on data management.

\section{References}

1. Imazio M. The post-pericardiotomy syndrome. Curr Opin Pulm Med. 2012;18: 366-74.

2. Imazio M, Brucato A, Rovere ME, Gandino A, Cemin R, Ferrua S, et al. Contemporary features, risk factors, and prognosis of the post-pericardiotomy syndrome. Am J Cardiol. 2011;108:1183-7.

3. Imazio M, Trinchero R, Brucato A, Rovere ME, Gandino A, Cemin R, et al. Colchicine for the prevention of the post-pericardiotomy syndrome (COPPS): a multicentre, randomized, double-blind, placebo-controlled trial. Eur Heart J. 2010;31:2749-54

4. van Osch D, Dieleman JM, Bunge JJ, van Dijk D, Doevendans PA, Suyker WJ, et al. Risk factors and prognosis of postpericardiotomy syndrome in patients undergoing valve surgery. J Thorac Cardiovasc Surg. 2017;153:878-85.e1.

5. Mott AR, Fraser CD, Kusnoor AV, Giesecke NM, Reul GJ, Drescher KL, et al. The effect of short-term prophylactic methylprednisolone on the incidence and severity of postpericardiotomy syndrome in children undergoing cardiac surgery with cardiopulmonary bypass. J Am Coll Cardiol. 2001;37:1700-6.

6. Nishimura RA, Fuster V, Burgert SL, Puga FJ. Clinical features and long-term natural history of the postpericardiotomy syndrome. Int J Cardiol. 1983;4: 443-54.

7. Lehto J, Kiviniemi T, Gunn J, Airaksinen J, Rautava P, Kytö V. Occurrence of postpericardiotomy syndrome: association with operation type and postoperative mortality after open-heart operations. J Am Heart Assoc. 2018;7:e010269.

8. Airaksinen KEJ, Grönberg T, Nuotio I, Nikkinen M, Ylitalo A, Biancari F, et al. Thromboembolic complications after cardioversion of acute atrial fibrillation: the FinCV (Finnish CardioVersion) study. J Am Coll Cardiol. 2013;62:1187-92.

9. Nuotio I, Hartikainen JEK, Grönberg T, Biancari F, Airaksinen KEJ. Time to cardioversion for acute atrial fibrillation and thromboembolic complications. JAMA. 2014;312:647-9.

10. Adler Y, Charron P, Imazio M, Badano L, Barón-Esquivias G, Bogaert J, et al. 2015 ESC guidelines for the diagnosis and management of pericardial diseases. Eur Heart J. 2015;36:2921-64.

11. Mehran R, Rao SV, Bhatt DL, Gibson CM, Caixeta A, Eikelboom J, et al. Standardized bleeding definitions for cardiovascular clinical trials: a consensus report from the bleeding academic research consortium. Circulation. 2011; 123:2736-47.

12. Lehto J, Gunn J, Karjalainen P, Airaksinen J, Kiviniemi T. Incidence and risk factors of postpericardiotomy syndrome requiring medical attention: the Finland postpericardiotomy syndrome study. J Thorac Cardiovasc Surg. 2015;149:1324-9.

13. van Osch D, Nathoe HM, Jacob KA, Doevendans PA, van Dijk D, Suyker WJ, et al. Determinants of the postpericardiotomy syndrome: a systematic review. Eur J Clin Invest. 2017;47:456-67.

14. Lehto J, Kiviniemi TO, Gunn J, Mustonen P, Airaksinen J, Biancari F, et al. Occurrence of postpericardiotomy syndrome admissions: a population-based registry study. Ann Med. 2016;48:28-33.

Key Words: postpericardiotomy syndrome, pericardium, thoracic surgery, mortality, adverse events 